\title{
HERNIA DEL ESPACIO DE PETERSEN: COMPLICACIÓN TARDÍA DEL BYPASS GÁSTRICO LAPAROSCÓPICO*
}

\author{
Drs. Jaime Zamarin M. ${ }^{1,2}$, Ismael Court R. ${ }^{1}$, Jorge Bravo L. ${ }^{1,2}$, Juan Eduardo Contreras P. ${ }^{1,2}$ \\ 1 Unidad de Cirugía Bariátrica y Metabólica, Clínica Santa María. \\ 2 Departamento de Cirugía Sede Oriente, Facultad de Medicina Universidad de Chile, Hospital del Salvador. \\ Santiago, Chile.
}

\begin{abstract}
\section{Petersen's space hernia: late complication of laparoscopic gastric bypass}

Introduction: Petersen's Space Hernia is a protrusion of intestinal loops through a defect between the alimentary loop and the transverse mesocolon that occur as a late complication of Gastric Bypass. Objective: To present our experience in the management of this entity, making emphasis on the difficulty of clinical diagnosis, the typical CT Scan findings and to suggest prevention and management strategies. Method: Case series study of patients with Petersen's Space Hernia after Laparoscopic Gastric Bypass. Clinical presentation, CT Scan findings and surgical results were analyzed. Results: 8 patients. Mean age 43 years. All presented al least 12 months after primary surgery, with unspecific abdominal pain. CT Scan most frequent findings were: Swirled mesentery, engorgement of mesentery vessels and the mushroom sign. Surgery was performed laparoscopically in the 8 patients. No bowel ischemia was found. In all cases, once reduced the protruded loops, complete closure of the defect with a running non absorbable suture was done. Conclusion: In patients with Gastric Bypass, presenting with abdominal pain, a high degree of suspicion must be kept about this entity. Clinical findings are unspecific and radiological study is crucial. When diagnosed on time bowel necrosis is avoided and the main surgical goal is to achieve a complete closure of the defect with non absorbable suture.
\end{abstract}

Key words: Petersen's space hernia, gastric bypass, bariatric surgery.

\section{Resumen}

Introducción: La Hernia del Espacio de Petersen es una complicación tardía del bypass gástrico, que ocurre por la protrusión de asas intestinales a través del defecto que se genera entre el asa alimentaria y el mesocolon transverso. Objetivo: Presentar nuestra experiencia en el manejo de esta patología, haciendo énfasis en la dificultad del diagnóstico clínico, los hallazgos más típicos de la Tomografía Computada (TC) y sugerir estrategias de prevención y manejo. Método: Estudio de una serie de casos con diagnóstico de Hernia del Espacio de Petersen posterior a bypass gástrico. Se analizó la presentación clínica, los hallazgos imagenológicos e intraoperatorios y los resultados del tratamiento quirúrgico. Resultados: 8 pacientes. Todos se presentaron luego de 12 meses de la cirugía primaria y la manifestación fue dolor abdominal de carácter

*Recibido el 24 de marzo de 2014 y aceptado para publicación el 28 de abril de 2014 .

Los autores no refieren conflictos de interés.

Correspondencia: Dr. Juan Eduardo Contreras P. juaneduardocontreras@gmail.com 
inespecífico. Los hallazgos más frecuentes de la TC fueron el "arremolinamiento" mesentérico, ingurgitación de los vasos mesentéricos y el "signo del hongo". La cirugía se realizó por vía laparoscópica en los 8 pacientes. No se evidenció isquemia intestinal. En todos los casos, una vez reducido el contenido herniado, se cerró el espacio con sutura continua de material no reabsorbible. No hubo complicaciones ni mortalidad. Conclusión: En los pacientes sometidos a bypass gástrico hay que mantener un alto grado de sospecha acerca de esta entidad. La clínica es inespecífica y el estudio imagenológico es fundamental. Pesquisado a tiempo se puede evitar la necrosis intestinal y el pilar fundamental del tratamiento es lograr un adecuado cierre del defecto con material no reabsorbible.

Palabras clave: Hernia del espacio de Petersen, bypass gástrico, cirugía bariátrica.

\section{Introducción}

La Hernia del Espacio de Petersen fue descrita por el cirujano alemán Walther Petersen ${ }^{1}$. Durante la era de la cirugía de úlcera péptica, era una entidad de presentación frecuente, sin embargo, con el ocaso de ésta, también cayó la incidencia de dicha complicación. Actualmente ha retomado importancia y cada vez se diagnostica más frecuentemente producto del crecimiento explosivo que ha tenido la cirugía bariátrica y puntualmente el bypass gástrico laparoscópico ${ }^{2}$.

Esta hernia se produce por la protrusión de las asas intestinales a través del defecto que se genera entre el asa alimentaria ascendida y el mesocolon transverso, cuando se realiza una reconstrucción en $\mathrm{Y}$ de Roux (Figura 1).

La presentación clínica es un desafío, ya que es totalmente inespecífica, caracterizada principalmente por dolor abdominal de características muy variables (puede ser cólico, constante, difuso o localizado, etc.) y sin elementos claros de obstrucción intesti$\mathrm{nal}^{3}$. Por esto es que el cirujano debe conocer esta entidad y siempre mantener un alto grado de sospecha acerca de su presencia, dado que un diagnóstico tardío puede resultar en necrosis intestinal ${ }^{4}$.

El objetivo de este estudio es presentar la experiencia de nuestro equipo en el manejo de esta patología, haciendo énfasis en la dificultad del diagnóstico clínico, los hallazgos más típicos de la TC y sugerir estrategias de prevención y manejo.

\section{Material y Método}

Estudio retrospectivo de una serie de casos de pacientes operados de hernia del espacio de Petersen, en Clínica Santa María, entre los años 2012 y 2013.

Los casos estudiados se incluyeron de acuerdo a los siguientes criterios: Pacientes sometidos a bypass gástrico laparoscópico en Clínica Santa María u otra institución y operados de hernia del espacio de Petersen por médicos del equipo de cirugía bariátrica, de acuerdo a protocolos prospectivos de manejo del equipo. Se excluyeron los pacientes operados en los que la causa de obstrucción fueran adherencias o bridas y cualquier otra hernia interna.

Se estudiaron variables demográficas (sexo y edad), tiempo de presentación desde la cirugía primaria, síntomas de presentación, hallazgos del examen físico, estudio imagenológico, abordaje y estrategia quirúrgica, complicaciones perioperatorias y estadía hospitalaria. Se contó con el consentimiento informado de los pacientes para obtener la información de los registros clínicos.

\section{Protocolo de manejo}

Para evitar la falla en el diagnóstico de esta entidad, se ha llevado a cabo un protocolo prospectivo de manejo, en que cualquier paciente portador de un bypass gástrico que se presenta con dolor abdominal inespecífico, pero sugerente de esta complicación (dolor abdominal intermitente postprandial o dolor

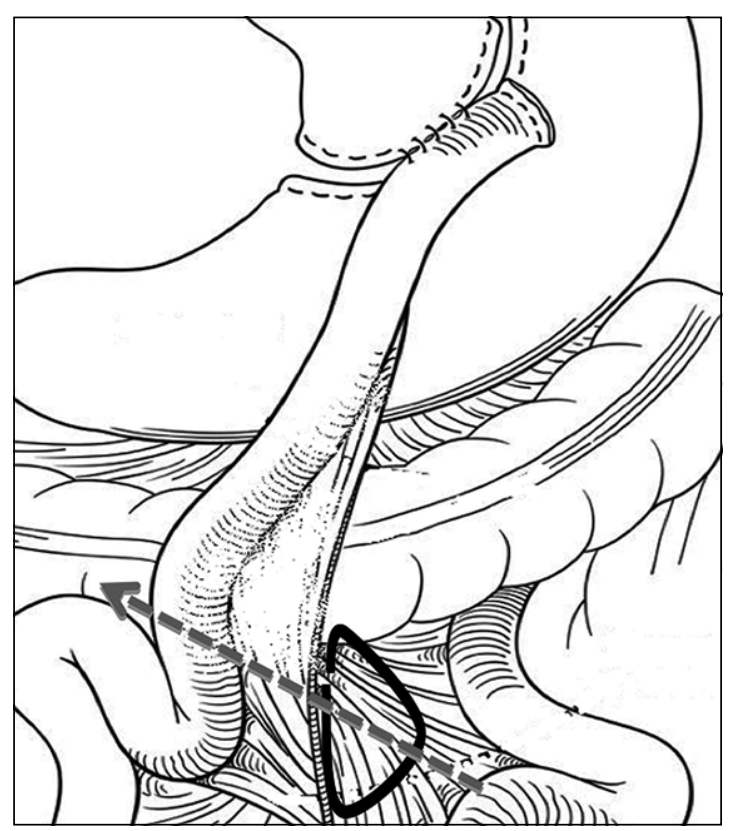

Figura 1. Espacio de Petersen. El semicírculo negro demuestra el espacio que se forma entre el asa alimentaria y el mesocolon transverso. La línea roja punteada señala el sentido por el que ocurre la protrusión del intestino. 
abdominal persistente sin una causa clara) y en el cual se ha descartado la presencia de un cólico biliar, colecistitis aguda, pancreatitis aguda y úlcera de boca anastomótica, es sometido a una TC de abdomen y pelvis.

\section{Resultados}

Entre los años 2012 y 2013 se operaron 8 pacientes de hernia del espacio de Petersen, con igual proporción entre géneros. Promedio de edad 43 años (rango: 22-52). Todos los pacientes presentaron el cuadro entre 12 a 24 meses de la cirugía primaria, con excepción de 1 caso que ocurrió en el octavo año postoperatorio. Característicamente en todos los casos hubo una adecuada baja de peso posterior al bypass gástrico y al momento en que se presentó la complicación el promedio de porcentaje de pérdida de exceso de peso (\%PEP) fue $85 \%$ (rango: $67-107 \%$ ). En 5 pacientes no fueron cerradas las brechas mesentéricas en la cirugía primaria, y no hay registro de los 3 casos restantes. La presentación clínica tuvo una gran variabilidad que se detalla en la Tabla 1. En todos los casos la TC demostró signos sugerentes de esta condición (Tabla 1), siendo lo más frecuente de encontrar la presencia de "arremolinamiento" del mesenterio, ingurgitación de los vasos mesentéricos y el "signo del hongo". Las Figuras 2 y 3 demuestran los hallazgos previamente señalados.

Todos los pacientes fueron operados por vía laparoscópica, sin necesidad de conversión a cirugía abierta. No se pesquisó necrosis intestinal en ningún caso. De los hallazgos intraoperatorios destaca la presencia en todos los casos de un exudado blanquecino de características quilosas en leve a moderada cuantía. Seis pacientes tenían el intestino delgado volvulado y congestivo, pasando de izquierda a derecha del paciente a través del espacio de Petersen y 2 casos presentaban intestino edematoso y congestivo reducido, con espacio de Petersen abierto, sin bridas ni otras hernias internas. Todos los pacientes evolucionaron sin complicaciones postoperatorias, no hubo mortalidad y el promedio de estadía hospitalaria fue de 2,2 días. Hasta la fecha de término de este estudio, no ha ocurrido recurrencia en ninguno de los casos operados.

\section{Estrategia quirúrgica}

Abordaje laparoscópico con el paciente en decúbito supino, en Fowler de $30^{\circ}$ y las extremidades inferiores en posición anatómica. El cirujano posicionado a la derecha del paciente junto con el camarógrafo y el ayudante a la izquierda del paciente. El pneumoperitoneo se realiza con técnica abierta para evitar lesiones intestinales secundarias a las adherencias postquirúrgicas. Los trócares posicionados triangulando hacia el hemi-abdomen superior-izquierdo (Figura 4). Se lleva a cabo una completa exploración abdominal identificando el Espacio de Petersen y otras posibles hernias internas o bridas y adherencias. Para identificar la hernia y su contenido, sistemáticamente se recorre el intestino delgado desde la gastroyeyuno anastomosis hasta la válvula ileocecal. Es característico que en la hernia del espacio de Petersen el intestino pasa de izquierda a derecha del paciente, por lo que la anastomosis yeyunoyeyunal queda ubicada a la derecha del asa alimentaria, el lado contrario al que se deja en la cirugía primaria. Todas las adherencias deben ser liberadas y luego retornar las asas intestinales a su posición original, realizando una tracción suave de éstas, para evitar el daño de la pared inflamada y friable. Se debe valorar la vitalidad del intestino y resecarlo según su condición. El último paso es el cierre completo del espacio de Petersen utilizando

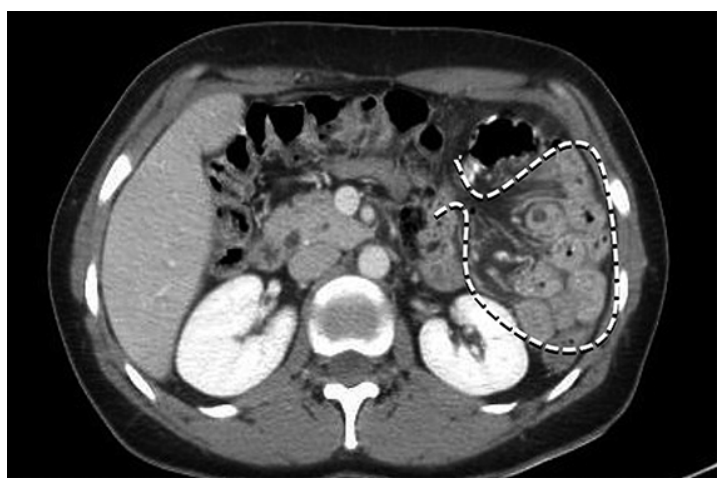

Figura 2. Signo del Hongo. La línea punteada demuestra la disposición típica que forma el mesenterio (tallo) y las asas intestinales aglomeradas (cabeza).

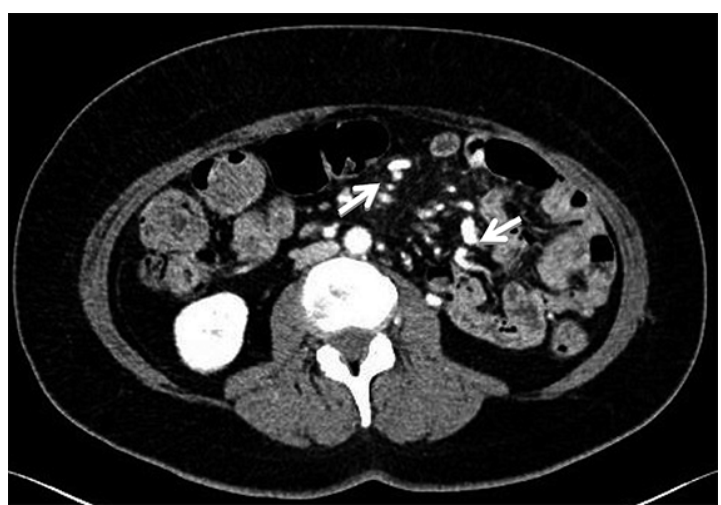

Figura 3. Ingurgitación de los vasos mesentéricos. Las flechas señalan vasos mesentéricos dilatados y tortuosos. 


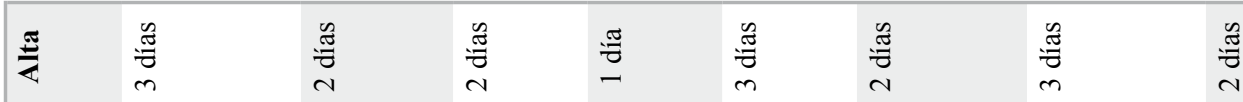

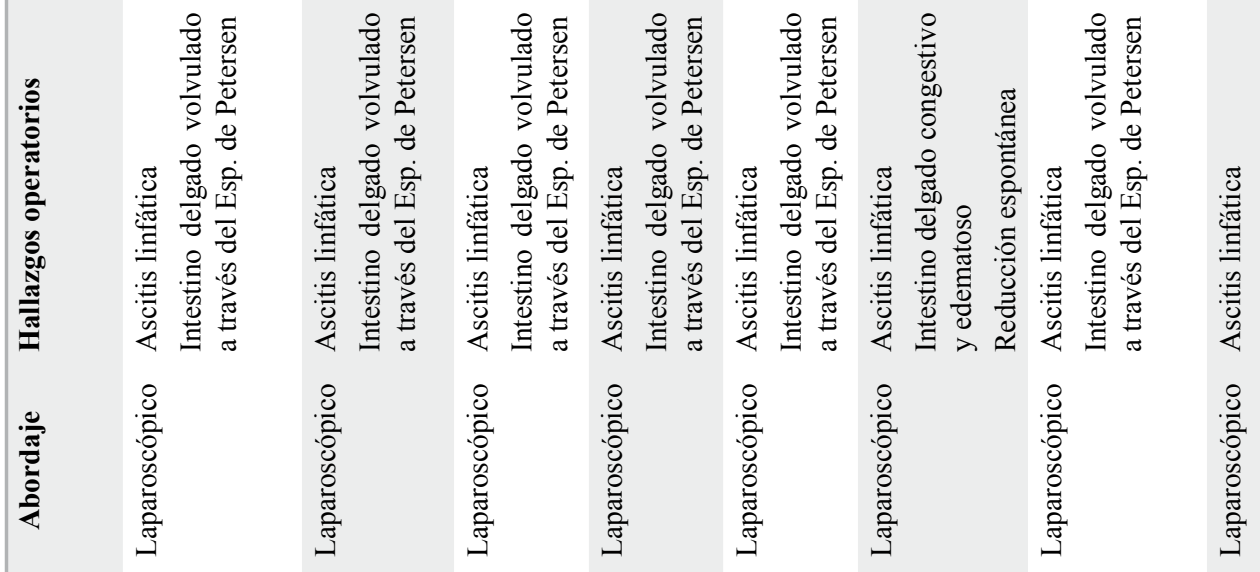

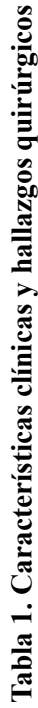

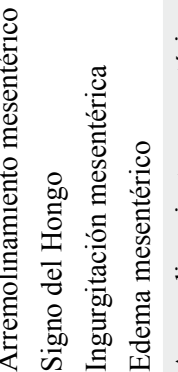

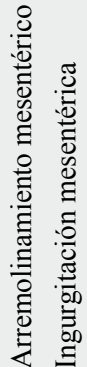
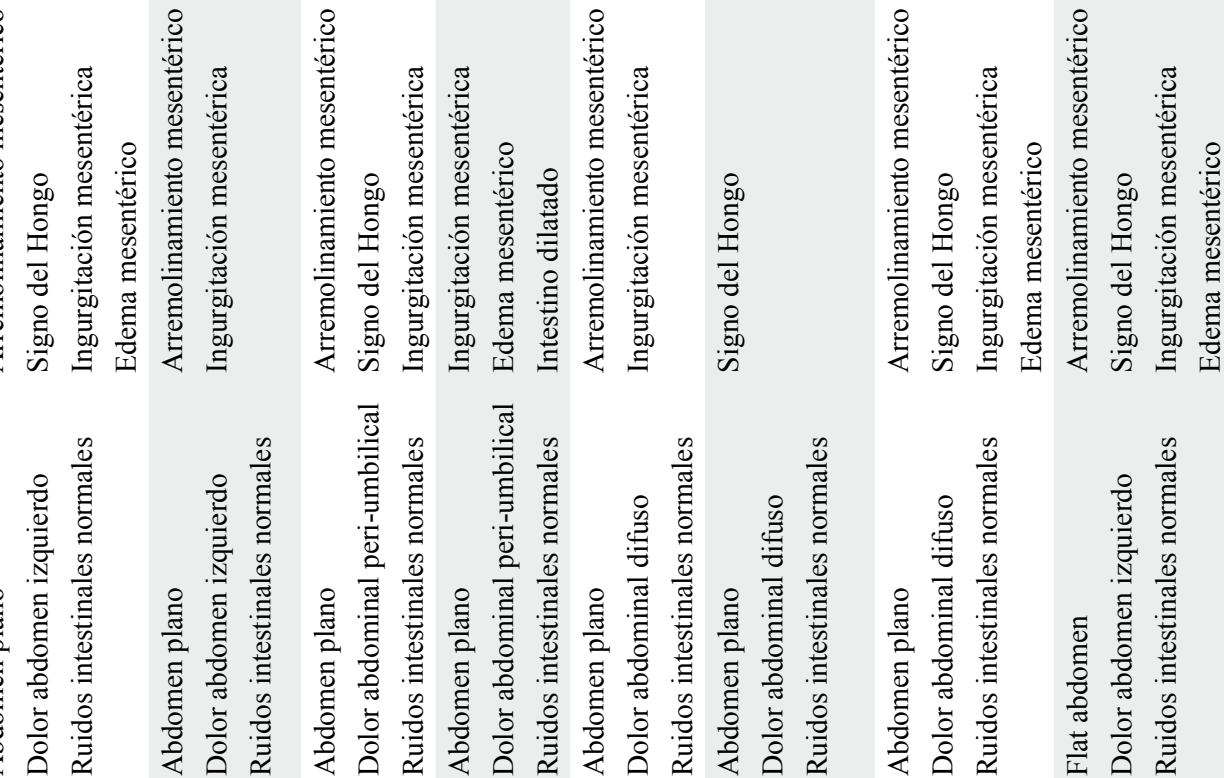

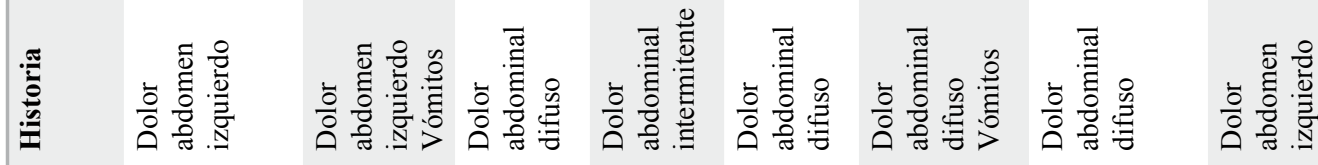

$\frac{\sqrt[T]{2}}{2}$

ڤे

ஸे

$\grave{2}$

ํํำ

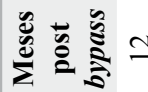

$\overline{2}$

$\approx$

$\because \quad 9$

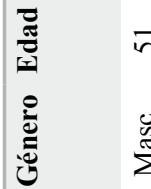

$\stackrel{f}{*}$

ง

กี

m

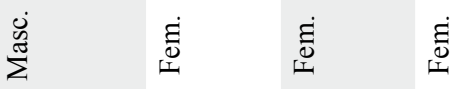

छ்

衰

$\frac{2}{6}$ 


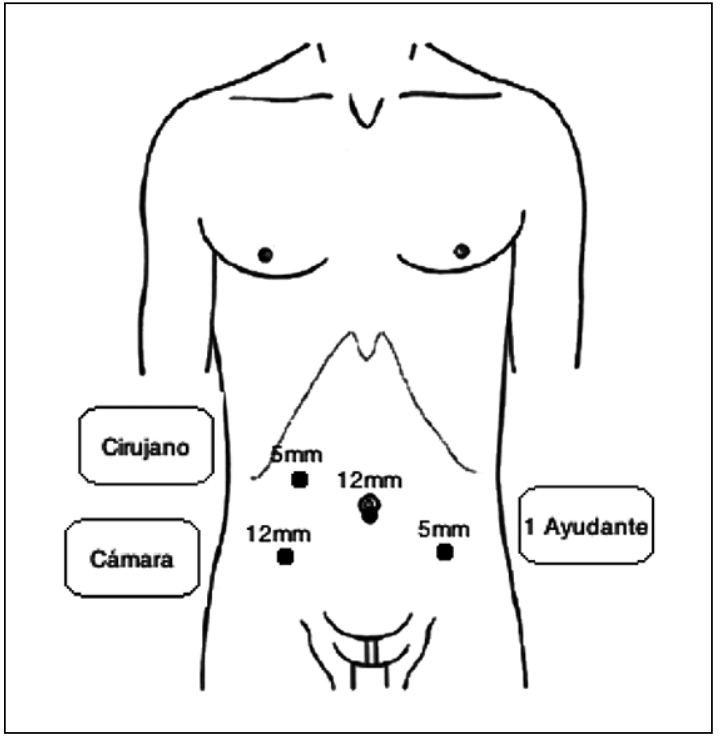

Figura 4. Posición del equipo quirúrgico y trócares.

una sutura continua con material no reabsorbible, siempre teniendo cuidado de no lesionar los vasos mesentéricos que se encuentran ingurgitados. De esa manera se evitan sangrados y la isquemia de la zona que irrigan dichos vasos.

\section{Discusión}

A pesar de todos los beneficios documentados de la cirugía laparoscópica, en el bypass gástrico se ha incrementado la tasa de obstrucción intestinal por hernias internas, lo que no ocurría con la técnica abierta, probablemente por el rol "protector" de las adherencias $^{5}$. La ausencia de éstas permite al intestino moverse libremente, y evita que el mesenterio se adhiera entre sí, quedando, por lo tanto, brechas mesentéricas abiertas ${ }^{6}$. Además, la cirugía abierta de la úlcera péptica habitualmente contemplaba el ascenso transmesocólico, tanto en reconstrucción tipo Billroth II como en Y de Roux, lo que anatómicamente haría menos probable la formación de una hernia interna. Es por esto que la incidencia de hernias internas aumentó de menos de $1 \%$ en cirugía abierta a 3-9\% en la era laparoscópica ${ }^{2,7,8}$.

Para prevenir esta complicación, hay autores que sugieren que el cierre de todas las brechas mesentéricas disminuiría su incidencia ${ }^{9-11}$, sin embargo, este es un tema controversial, porque otros autores creen que la baja masiva de peso y de grasa intraabdominal que ocurre en el postoperatorio puede generar un aumento del tamaño de los defectos mesentéricos pese a un adecuado cierre en la cirugía primaria $^{12}$. Pese a lo anterior, la evidencia actual tiende a demostrar que si bien el cierre de los defectos mesentéricos en la cirugía primaria no previene totalmente la aparición de hernias internas, este gesto disminuye su incidencia a la mitad ${ }^{8}$.

La presentación clínica aguda de la hernia del espacio de Petersen varía ampliamente, siendo frecuente encontrar dolor abdominal difuso o localizado (principalmente en el hemiabdomen izquierdo), constante o intermitente, asociado o no a vómitos ${ }^{13}$. Además, se puede presentar como un cuadro crónico, con dolor recurrente postprandial, que obliga al paciente a consultar reiteradamente, deteriorando su calidad de vida y llevando al médico a variados estudios sin resultados concluyentes, siendo a veces calificados como portadores de cuadros digestivos funcionales e incluso derivados al psiquiatra.

En este contexto clínico, y sin una clara explicación (descartados los cuadros biliares, pancreatitis aguda y la úlcera de boca anastomótica), la TC de abdomen y pelvis es mandatoria, ya que permite realizar el diagnóstico precozmente. Tanto el radiólogo como el cirujano deben buscar los signos tomográficos típicos de esta patología, como el arremolinamiento mesentérico, que se aprecia como un remolino o un aspecto de tornado, de los vasos y grasa de la raíz del mesenterio, que se hace especialmente evidente cuando se recorren en forma dinámica los cortes de la tomografía. Se debe ser cuidadoso, ya que esto puede verse en pacientes sanos, por lo que siempre debe valorarse en el contexto del paciente y de otros hallazgos de las imágenes, como el signo del hongo, en el que se aprecia una disposición fungiforme, cuyo tallo está compuesto por el mesenterio y la cabeza por las asas intestinales aglomeradas. Además es frecuente encontrar ingurgitación de los vasos mesentéricos, los que aparecen dilatados y tortuosos. Otros hallazgos a conocer son la disposición del intestino delgado por detrás de la arteria mesentérica superior, la presencia de la anastomosis yeyunoyeyunal a la derecha del asa alimentaria y el desplazamiento hacia anterior y a derecha del ligamento de Treitz ${ }^{14-16}$.

Cuando esta complicación se presenta en el contexto de un cuadro de obstrucción intestinal o con dolor intenso, es necesaria la exploración quirúrgica de urgencia ${ }^{12}$. Como se presentó previamente, todos los pacientes deberían ser explorados y tratados por vía laparoscópica, realizando la conversión a cirugía abierta si el equipo médico no cuenta con experiencia laparoscópica o si por esta vía no es posible corregir el defecto o las consecuencias de éste.

Los principios quirúrgicos en esta cirugía son tres: La correcta identificación de las brechas mesentéricas, por lo que el conocimiento anatómico de los defectos del mesenterio postoperatorio es crucial 


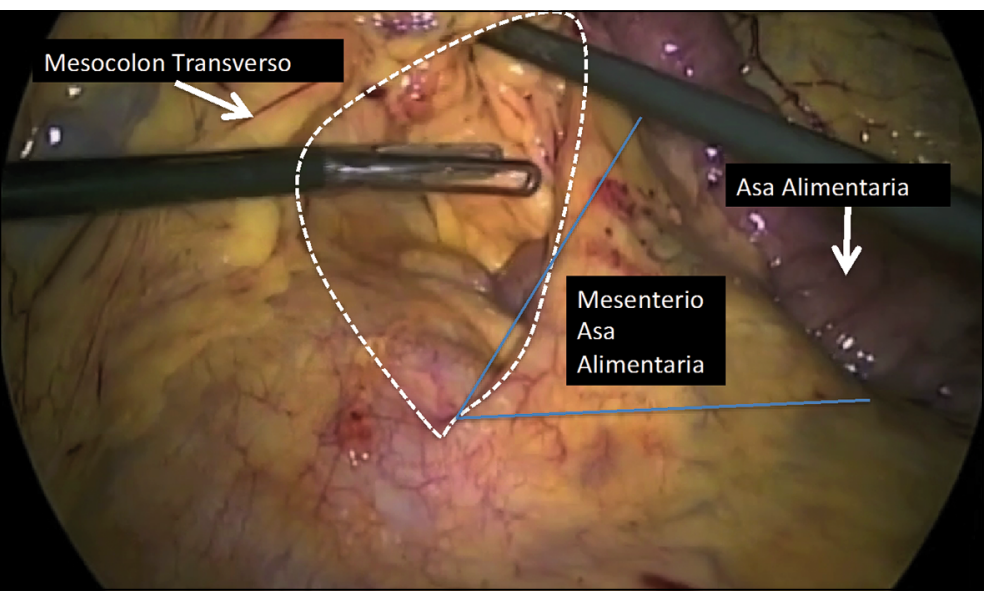

Figura 5. Espacio de Petersen (visión intraoperatoria). La figura muestra el espacio visto desde la derecha del paciente, que es la posición para el cierre. La línea punteada muestra el espacio entre el mesenterio del asa alimentaria y el mesocolon transverso.

(Figura 5). La reducción suave del intestino herniado, evaluando su vitalidad. Y por último, el cierre completo de todos los defectos mesentéricos con sutura continua de material no reabsorbible.

Los buenos resultados mostrados en este estudio pertenecen a un grupo de pacientes manejados por un grupo experto en el tema, por lo que quizás éstos no se pueden extrapolar a cualquier grupo quirúrgico. Del punto de vista metodológico, la recopilación de datos es retrospectiva, pero no por esto pierde valor, dado que no intenta responder a una pregunta, sino más bien presentar una complicación de baja frecuencia, detallando sus características clínicas. Además, este reporte es el primero en la literatura chilena acerca de esta entidad y propone una conducta de estudio y manejo quirúrgico basado en buenos resultados.

\section{Conclusión}

Todos los cirujanos, especialmente los que no realizan cirugía bariátrica, deben conocer y estar atentos a esta complicación tardía de bypass gástrico laparoscópico, manteniendo un alto grado de sospecha en el paciente con dolor abdominal y sin una clara causalidad. La TC es una excelente herramienta diagnóstica en este contexto, sin embargo, el radiólogo y el cirujano deben saber qué elementos buscar.

A pesar de que aún existe controversia, la evidencia sugiere que cerrando los defectos mesentéricos se disminuye la posibilidad de desarrollar una hernia interna, por lo que se aconseja seguir esta conducta. Cuando la complicación se presenta, el cierre completo de las brechas mesentéricas, utilizando una sutura continua de material no reabsorbible, es mandatorio.

\section{Referencias}

1. Petersen $\mathrm{W}$. Ueber darmveschlingung nach der gastroenterostomie. Arch Klin Chir. 1900;62:94-114.

2. Higa D, Ho T, Boone KB. Internal hernias after laparoscopic Roux-en-Y gastric bypass: incidence, treatment and prevention. Obesity Surgery 2003;13:350-4.

3. Paroz A, Calmes JM, Giusti V, Suter M. Internal hernia after laparoscopic Roux-en-Y gastric bypass for morbid obesity: a continuous challenge in bariatric surgery. Obesity Surgery 2006;16:1482-7.

4. Reiss J, Garg V. Bowel gangrene from strangulated Petersen's space hernia after gastric bypass. J Emerg Med. 2014;46:e31-4.

5. Higa K, Boone K, Arteaga González I, López-Tomassetti Fernández E. Mesenteric closure in laparoscopic gastric bypass: surgical technique and literature review. Cirugía Española 2007;82:77-88.

6. Parakh S, Soto E, Merola S. Diagnosis and management of internal hernias after laparoscopic gastric bypass. Obesity Surgery 2007;17:1498-502.

7. Podnos YD, Jiménez JC, Wilson SE, Stevens CM, Nguyen NJ. Complications after laparoscopic gastric bypass: a review of 3464 cases. Arch Surg 2003;138:957-61.

8. Capella RF, Iannace VA. Capella JF. Bowel obstruction after open and laparoscopic gastric bypass surgery for morbid obesity. J Am Coll Surg. 2006;203:328-35.

9. Brolin R, Venkata K. Impact of complete mesenteric closure on small bowell obstruction and internal mesenteric hernia after laparoscopic Roux-en-Y gastric bypass. Surg Obes Relat Dis. 2013:850-5.

10. Champion JK, Williams M. Small bowel obstruction and internal hernias after laparoscopic Roux-en-Y gastric bypass. Obes Surg. 2003;13:596-600.

11. Higa KD, Boone KB, Ho T. Complications of the laparoscopic Roux-en-Y gastric bypass: 1,040 patients-what have we learned? Obes Surg. 2000;10:509-13. 
12. Faria G, Preto J, Pimenta T. Petersen space hernia: a rare but expanding diagnosis. Int J Surg Case Rep. 2011;2:140-3.

13. Aghajani E, Jacobsen H, Gislason H. Internal hernia after gastric bypass: A new and simplified technique for laparoscopic primery closure of the mesenteric defects. J Gastrointest Surg. 2011.16:641-5.

14. Carrera C, De Luca S, Andrade F. Hernia de Petersen. Complicación del bypass gástrico: Hallazgos to- mográficos. Rev Argent Radiol. [online]. 2012;3:2414.

15. Meralli H, Miller C, Ghosh A. Importance of CT in evaluating internal hernias after Roux-en-Y gastric bypass surgery. J Radiol Case Rep. 2009;3:34-7.

16. Srikanth MS, Keskey T, Fox SR, Oh KH, Fox ER, Fox KM. Computed tomography patterns in small bowel obstruction after open distal gastric bypass. Obesity Surgery 2004;14:811-22. 